\title{
Experiencia didáctica de una adaptación de 3 famosos programas de TV: First Dates, MásterChef y Pekín Express al aula universitaria
}

\author{
Arufe Giráldez, Víctor \\ Unidad de Investigación del Deporte Escolar, Educación Física y Psicomotricidad. \\ Departamento Didácticas Especificas. Métodos de Investigación y Diagnóstico en Educación \\ Facultad de Ciencias de la Educación. Universidade da Coruña.
}

\section{RESUMEN}

El aula universitaria no puede estar ajena a lo que sucede en la sociedad y tampoco a los gustos y preferencias de ocio de los estudiantes. Los docentes deben aprovechar las herramientas que les ofrece la sociedad para poder crear un clima cálido en el aula, que favorezca el aprendizaje de los contenidos y la adquisición de múltiples competencias de cada materia. Se presentan tres experiencias didácticas realizadas con alumnado universitario basadas en la adaptación del formato de tres famosos programas de televisión con fines educativos y didácticos. Se utilizaron versiones adaptadas del programa First Dates, MásterChef y Pekín Express a fin de poder trabajar competencias y contenidos establecidos en las guías docentes de las tres materias. Para medir el impacto que produjeron estas actividades didácticas sobre la satisfacción del alumnado y su motivación hacia el estudio de los contenidos, se pasó un cuestionario anónimo una vez finalizada la experiencia. Los resultados confirman una gran satisfacción del alumnado mostrándose muy de acuerdo con las actividades y señalando que mejoraron su motivación para el estudio de la materia y aprendizaje de los contenidos.

PALABRAS CLAVE: Gamificación, programas televisión, Másterchef, First dates y Pekín Express. 


\section{CITA RECOMENDADA:}

Arufe Giráldez, V. (2019): Experiencia didáctica de una adaptación de tres famosos programas de TV: First Dates, MásterChef y Pekín Express al aula universitaria. En De la Torre Fernández, E. (ed.) (2019). Contextos universitarios transformadores: construíndo espazos de aprendizaxe. III Xornadas de Innovación Docente. Cufie. Universidade da Coruña. A Coruña (pág. 99-116).

DOl capítulo: https://doi.org/10.17979/spudc.9788497497121.099

DOl libro: https://doi.org/10.17979/spudc.9788497497121

\section{ABSTRACT}

The university classroom cannot be oblivious to what is happening in society, and neither to the preferences of the students. Teachers must take the opportunity to take advantage of the tools offered by society to enrich and create a warm atmosphere in the classroom, which favours the learning of contents and the acquisition of multiple skills in each subject. It presents a didactic experience with university students based on the adaptation of the format of three famous television programmes for educational and didactic purposes. Adapted versions of the First Dates, MasterChef and Beijing Express programmes were used in order to work with the contents established in the teaching guides for the three subjects. In order to measure the impact of these teaching activities on student satisfaction and their motivation to study the contents, an anonymous questionnaire was passed on after the experience was completed. The results confirm a great satisfaction of the students, who were very satisfied with the activities and indicated that they improved their motivation to study the subject and learn the contents.

KEY WORDS: Gamification, TV programmes, MásterChef, First dates and Pekin Express. 


\section{INTRODUCCIÓN}

Las últimas tendencias en el ámbito de la innovación educativa otorgan una gran importancia al saber cómo aprende el cerebro y cómo captar la atención del alumnado. Así es como la neuroeducación, término que ha cobrado fuerza en los últimos años, aboga por despertar la curiosidad del alumnado, favorecer el interés, el movimiento, utilizando metodologías activas donde el alumnado sea el principal protagonista del proceso de su formación (Mora Teruel, 2014).

El aula universitaria debe favorecer el aprendizaje de contenidos y ser un medio para conseguir trabajar diferentes valores y potenciar el capital humano del alumnado. El objetivo de cualquier docente debe ser que el alumnado ame y disfrute de su materia, y en último caso intentar que no la odie (Arufe Giráldez, 2018). Para conseguir esto, los docentes tienen que utilizar estrategias didácticas que aseguren unos niveles altos de motivación y que aprender sea un disfrute. Es difícil conseguirlo en algunas materias donde la carga de contenidos teóricos es elevada, pero seguramente a través del juego podamos mejorar los niveles de motivación del alumnado. El ser humano siempre disfrutó con el juego, cuando nacemos el juego es el mejor medio de aprendizaje, jugamos a lo largo de toda nuestra vida, solo que cambia la forma de jugar y el tipo de juego. Así es como surge el concepto de gamificación en el aula, entendido como una forma divertida y amena de trabajar los contenidos y competencias de las diferentes materias, siguiendo los principios que rigen el juego y realizando en todo momento una planificación de las actividades, control y seguimiento y vinculación completa con las competencias y resultados de aprendizaje previamente establecidos en las guías docentes y programaciones del docente. Todo ello con el fin de mejorar la motivación de los estudiantes hacia el estudio de la materia y comprensión de los contenidos.

La gamificación debe acaparar la atención de más investigaciones y establecer y concretar todo lo que conlleva este fenómeno (Deterding, Khaled, Nacke, \& Dixon, 2011).

Daniel Parente define una precisa arquitectura funcional dentro del proceso de gamificación, a cuyos elementos que la integran debemos prestar especial atención a la hora de elaborar 
propuestas de gamificación. Estos elementos son: la actividad, el contexto, las competencias y habilidades, la gestión y supervisión y la mecánica y los elementos del juego (Contreras Espinosa \& Eguia, 2016).

Aprovechando el boom mediático de numerosos programas de televisión y sabiendo que mucho alumnado consume programas de televisión en su tiempo de ocio, podemos incorporar al aula algunos de los programas más famosos vistos por los jóvenes universitarios y realizar pequeñas adaptaciones en su formato para conseguir nuestros fines didácticos.

En este trabajo, se presentan tres experiencias didácticas realizadas en el aula universitaria implementando tres famosos programas de televisión con sus correspondientes adaptaciones para fines didácticos.

\section{DESCRIPCIÓN DE LA EXPERIENCIA}

Aunque son experiencias didácticas diferentes todas ellas tienen un mismo hilo conductor, la utilización de diversos programas de televisión en el aula.

Para facilitar la comprensión de esta experiencia se establecen en el artículo tres grandes apartados con la descripción de cada una de ellas.

La Tabla 1 recoge información sobre las materias donde se realizó cada una de las experiencias didácticas.

\begin{tabular}{|c|c|c|c|c|}
\hline Experiencia & \multicolumn{2}{c}{ Titulación } & Curso & \multicolumn{2}{c|}{ Materia } & Alumnado \\
\hline First Dates & Grado de Educación Infantil & $2^{0}$ & $\begin{array}{c}\text { Didáctica de la Expresión } \\
\text { Corporal }\end{array}$ & 60 \\
\hline Máster Chef & Grado de Educación Primaria & $3^{0}$ & $\begin{array}{c}\text { Expresión Corporal y } \\
\text { Adaptaciones Curriculares } \\
\text { Educación Psicomotriz y } \\
\text { Habilidades Motrices } \\
\text { Básicas }\end{array}$ & 60 \\
\hline Pekín Express & Grado de Educación Infantil & $4^{0}$ & 45 \\
\hline
\end{tabular}

Tabla 1. Materias en las que se llevó a cabo la experiencia didáctica con programas de T.V. 


\section{Experiencia 1. Aplicación didáctica del programa de televisión First Dates}

Esta experiencia está basada en el famoso programa de televisión emitido por Cuatro TV y denominado First Dates. El programa real consiste en un lugar de encuentro para personas que busquen pareja 0 deseen encontrar a una persona que tenga, o no, sus mismas afinidades y gustos para establecer una buena sintonía y disfrutar de la vida conjuntamente. El lugar es un restaurante adaptado para la grabación continua de las conversaciones de quienes comparten una cena íntima para conocerse mejor y posteriormente decidir ante la cámara si inician un camino conjunto de proyecto de vida 0 si de lo contrario no encontraron a esa persona que buscaban.

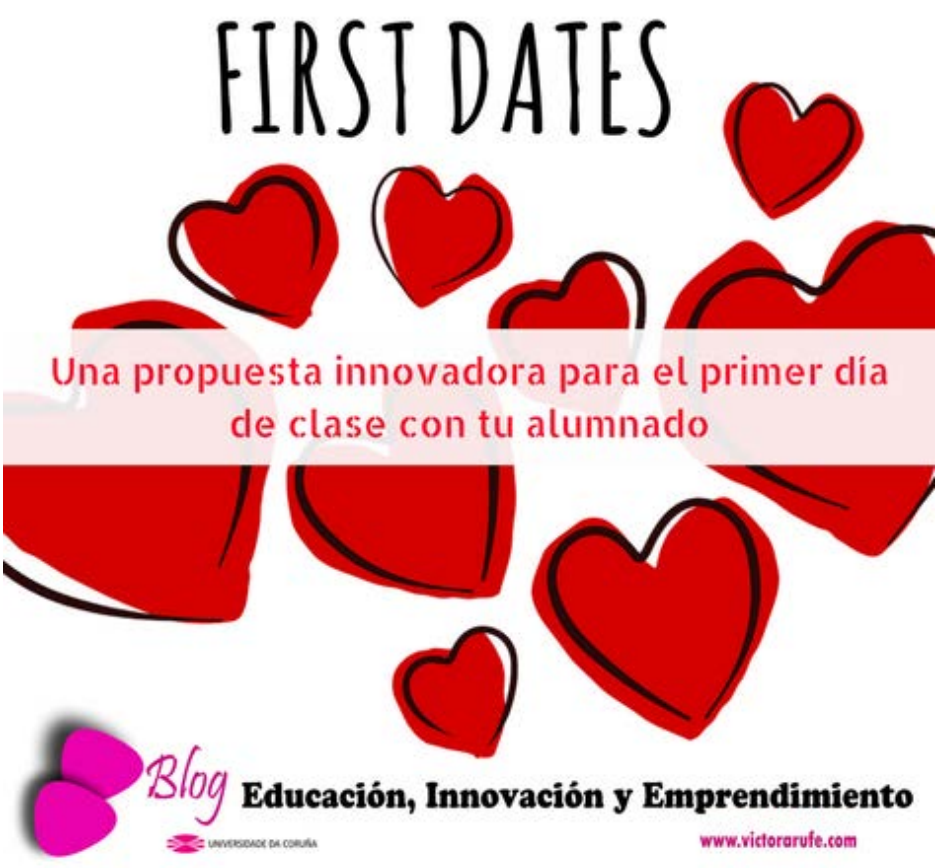

Imagen 1. Portada de la presentación en powerpoint de la actividad realizada en el aula

Para la experiencia llevada al aula, se adaptó el contenido del programa para que pudiera servir en el primer día de clase como un icebreakers o rompehielos. Generalmente, el primer día de 
clase mucho alumnado no se conoce entre si, especialmente en materias optativas o materias del primer año de carrera. Incluso esta iniciativa, podría ser válida para cualquier materia y año de carrera, dado que en muchas ocasiones el alumnado establece un pequeño círculo de amigos sin conocer más a fondo a otros compañeros/as.

La experiencia tiene como objetivo facilitar un clima cálido donde todos los alumnos/as conozcan un poco más el carácter, gustos y las aficiones de sus compañeros, a fin de poder trabajar la cohesión grupal y establecer nuevos círculos de amistades, mejorando así el trabajo en equipo "Team Building".

Para conseguir esto, el docente elabora una pequeña ficha en tamaño A4 o A2 e incorpora una serie de apartados que buscan conseguir información sobre gustos, preferencias, carácter, planes de futuro... del alumnado, sin desvelar datos relativos a nombre y apellidos 0 descripción física.

El profesor solicita a los estudiantes que cubran ese papel de forma completamente sincera. Cuando lo tengan cubierto, harán un avión de papel que lanzarán aleatoriamente a cualquier lugar de la clase cuando el profesor diga ya. En ese momento, cada estudiante cogerá un avión que cayese cercano a él y leerá la descripción de la persona que se esconde detrás de esas palabras. Todos intentarán adivinar quién es el estudiante propietario de ese avión.

En mi blog relato con más detalle cómo fue ese primer encuentro cuando le digo al alumnado el primer día de clase si les apetece jugar a First Dates..."miradas desconcertadas, caras sonrientes, incertidumbre..., hasta que le explicas que realizaremos un pequeño juego de presentación y conocimiento"(Arufe Giráldez, 2017a).

El objetivo no es otro que conocerse entre todos y romper el hielo. No se puede concebir un proceso de enseñanza-aprendizaje sin saber a quién se tiene delante, sin conocer a los alumnos/as.

La experiencia cumple varios objetivos:

- Permitir al alumnado conocerse un poco más entre ellos, dando la oportunidad de crear vínculos afectivos relativos a intereses, gustos o aficiones. 
- Conseguir que el docente conozca un poco más al grupo que tiene en el aula a fin de poder mejorar las estrategias educativas y la calidad docente, captar su atención y despertar su interés.

- Facilitar la desinhibición del alumnado y crear un clima distendido que pueda favorecer futuros aprendizajes.

En la Tabla 2, se exponen las competencias alcanzadas con esta materia que han sido establecidas en la guía docente de la misma.

\section{Competencias alcanzadas}

Saber utilizar el juego como recurso didáctico, así como diseñar actividades de aprendizaje basadas en principios lúdicos

Expresarse correctamente, tanto de forma oral como escrita, en las lenguas oficiales de la comunidad autónoma

Trabajar de forma colaborativa

Autonomía en el aprendizaje

Capacidad de análisis y síntesis

Comunicación oral y escrita en la lengua materna

Capacidad para relacionarse positivamente con otras personas

Tabla 2. Competencias establecidas en la Guía Docente de la materia que fueron trabajadas durante la realización de esta actividad

\section{Experiencia 2. Aplicación didáctica del programa de televisión MásterChef}

Esta segunda experiencia está basada en el famoso programa de televisión que hace referencia a la gastronomía y saber cocinar exquisitos platos en un tiempo determinado previamente y con unos ingredientes elegidos por los propios participantes. 


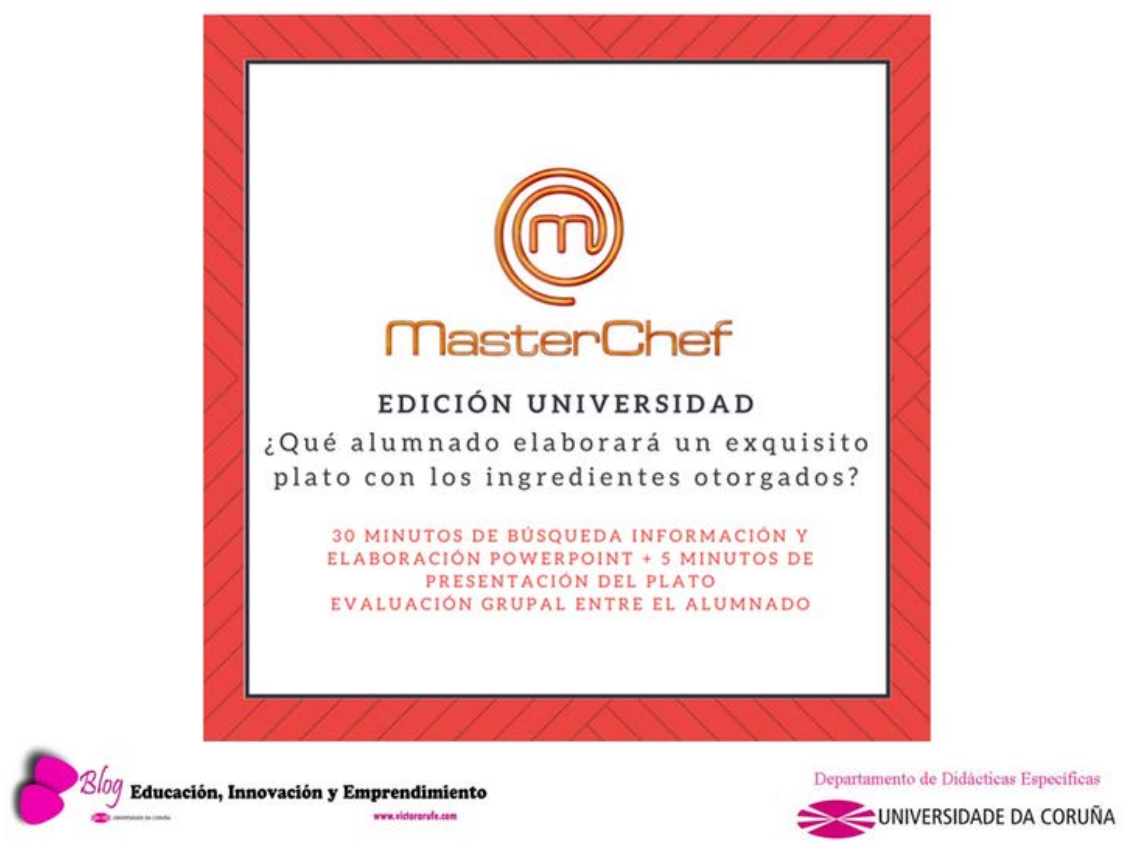

Imagen 2. Portada de la presentación en powerpoint realizada en el aula para el conocimiento por parte del alumnado de la actividad

Se realizó en el aula para trabajar diferentes contenidos de la materia y conseguir diversas competencias establecidas en la misma.

La mecánica es la siguiente: el docente forma grupos de 4-5 alumnos e incorpora contenidos a trabajar en sobres de papel cerrados sin que ningún alumno sepa el contenido que hay en cada sobre.

Todos los sobres se colocarán en una mesa dentro del aula y en otra mesa aparte se colocará el material didáctico correspondiente a cada uno de los contenidos que engloban los sobres. Cuando el docente diga ya, un representante de cada grupo deberá acudir rápidamente a coger un sobre, abrirlo para ver el contenido que le tocó desarrollar (plato a cocinar) y, posteriormente acudirá a la otra mesa para coger el material didáctico (ingredientes) correspondiente a ese plato y necesario para cocinarlo. Es importante hacer notar al alumnado 
que si se equivoca cogiendo el material didáctico perderá tiempo y tendrá que devolver esos apuntes y cambiarlos por otros.

Una vez que ya sabe el plato y tiene los ingredientes, se incorporará con el resto de los compañeros/as de su grupo y tendrán de 30 minutos para confeccionar un powerpoint 0 similar con toda la información necesaria para explicar a sus compañeros/as y exponer sus ideas y contenidos. Los estudiantes pueden completar la información añadiendo otras fuentes de información, como búsqueda de libros en la biblioteca, artículos técnico-científicos en bases de datos, visitas a webs de información fiable, etc.

Antes de finalizar los 30 minutos, el representante de cada grupo deberá entregar al profesor una memoria USB con su trabajo. Para dar más emoción a este juego, el profesor pondrá en la pantalla un temporizador que marque el tiempo restante y música de fondo ideal para trabajar.

El alumnado trabaja de esta forma diferentes competencias (Tabla 3) y contenidos (Tabla 4). Algunas de ellas son: competencias digitales y lingüísticas, capacidad de ser crítico con la información manejada, de síntesis de información y de trabajo en equipo, y otros muchos valores que van intrínsecos a esta actividad. Pese a no ser la primera vez que realizaba esta experiencia, sí que fue la primera vez que la grabé y en mi blog se puede ver un pequeño vídeo resumen (Arufe Giráldez, 2017b).

\begin{tabular}{|c|}
\hline Competencias alcanzadas \\
\hline $\begin{array}{c}\text { Identificar dificultades de aprendizaje, informarlas y colaborar en su tratamiento. } \\
\text { Promover el trabajo cooperativo y el trabajo y esfuerzo individual }\end{array}$ \\
\hline $\begin{array}{c}\text { Promover acciones de educación en valores orientadas a la preparación de una ciudadanía activa y democrática } \\
\text { Desarrollar y evaluar contenidos del currículo mediante recursos didácticos apropiados y promover las } \\
\text { competencias correspondientes a los estudiantes. }\end{array}$ \\
Adquirir un conocimiento práctico del aula y de su gestión \\
\hline $\begin{array}{c}\text { Conocer y aplicar los procesos de interacción y comunicación en el aula y dominar las destrezas y habilidades } \\
\text { sociales necesarias para fomentar un clima de aula que facilite el aprendizaje y la convivencia. }\end{array}$ \\
\hline Participar en la actividad docente y aprender a saber hacer, actuando y reflexionando desde la práctica. \\
\hline Aprender a aprender. \\
\hline Aplicar un pensamiento crítico, lógico y creativo. \\
\hline Comportarse con ética y responsabilidad social como ciudadano y como profesional. \\
\hline Capacidad de comprensión de los distintos códigos audiovisuales y multimedia y manejo de las herramientas \\
informáticas.
\end{tabular}


Capacidad de selección, de análisis, de evaluación y de utilización de distintos recursos en la red y multimedia.

Capacidad para trabajar en equipo de forma cooperativa, para organizar y planificar el trabajo, tomando decisiones y resolviendo problemas, tanto de forma conjunta como individual.

Capacidad para utilizar diversas fuentes de información, seleccionar, analizar, sintetizar y extraer ideas importantes y gestionar la información.

Capacidad crítica y creativa en el análisis, planificación y realización de tareas, como fruto de un pensamientos flexible y divergente

Compromiso ético para el ejercicio de las tareas docentes.

Utilizar las herramientas básicas de la tecnología de la información y comunicación necesarias para el ejercicio de su profesión y para el aprendizaje a lo largo de la vida

Capacidad de análisis y de autoevaluación del propio trabajo como del trabajo en grupo

Tabla 3. Competencias establecidas en la Guía Docente de la materia que fueron trabajadas durante la realización de esta actividad

\section{Contenidos abordados}

Descripción de las principales discapacidades psíquicas. Características y manifestaciones de las distintas discapacidades psíquicas.

Descripción de las principales discapacidades sensoriales. Características y manifestaciones de las distintas discapacidades sensoriales

Descripción de las principales discapacidades físicas. Características y manifestaciones de las distintas discapacidades físicas.

Tabla 4. Contenidos establecidos en la Guía Docente de la materia que fueron trabajados durante la realización de esta actividad

\section{Experiencia 3. Aplicación didáctica del programa de televisión Pekín Express}

La tercera experiencia consistió en adaptar el programa de TV Pekín Express a las necesidades de trabajo educativo en el aula. El programa consiste en una competición por grupos de personas que deben ir completando unas misiones encomendadas por la dirección y con un presupuesto y recursos limitados. 


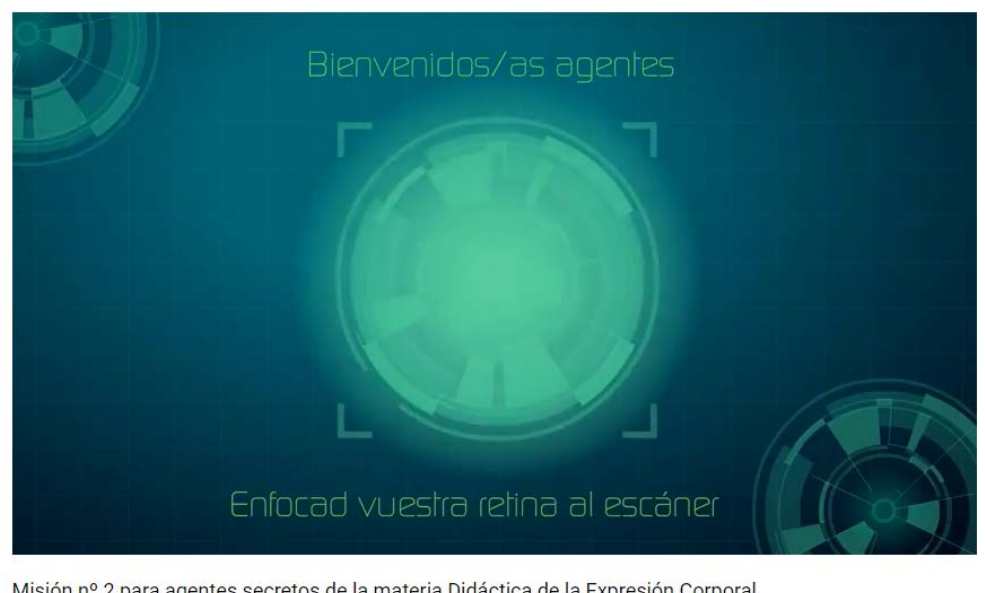

Imagen 3. Imagen extraída del vídeo de una de las misiones secretas publicado en el canal de youtube del docente

Para llevarlo al aula, se solicita al alumnado que formen grupos de trabajo de 5-6 personas y se asignen un nombre de guerra. El profesor les indica que desde ese momento son agentes secretos suyos y tendrán que ir realizando cada 15 días unas misiones que se difundirán a través del canal de youtube del docente.

En total son 5 misiones las que tienen que realizar a lo largo del cuatrimestre, todas ellas relacionadas con contenidos de la materia establecidos en su guía docente.

Los grupos se enterarán de las nuevas misiones a través de un mensaje que les llegará a todos al mismo tiempo por la plataforma oficial de Moodle de la Universidade da Coruña. Estas misiones se pueden enviar a cualquier hora del día y cualquier día de la semana, por lo que deberán estar atentos, pues se valora el tiempo que invierte el alumnado en realizarla.

Cuando un grupo finaliza la misión, debe entregarla al profesor en su despacho o por el medio que se requiera. En ese momento el alumnado conocerá el puesto en el que ha quedado y se asignará una cantidad de puntos según su puesto.

Al final de la materia, ganará el grupo que más puntos consiguiese. El premio, anunciado desde el primer día de clase, será un desayuno completo con el docente, donde se 
aprovechará la ocasión para hablar de manera distendida sobre la materia, debatir sobre la docencia realizada, contenidos que más le gustó al alumnado, etc.

Son varios los objetivos de esta actividad,

- Fomentar el trabajo en equipo.

- Conseguir desarrollar y trabajar de forma lúdica los contenidos de la materia.

- Potenciar el trabajo autónomo del alumnado, así como la toma de decisiones y autonomía.

- Consolidar los conocimientos adquiridos en el aula.

- Conocer la realidad externa al centro educativo.

- Potenciar la constancia y el esfuerzo.

En la Tabla 5, se recogen las competencias alcanzadas y que están reflejadas en la Guía Docente de la materia.

\section{Competencias alcanzadas}

Conocer la dimensión pedagógica de la interacción con los iguales y adultos y saber cómo promover la participación en actividades colectivas, trabajo cooperativo y esfuerzo individual

Saber cómo informar a otros especialistas profesionales para abordar la colaboración del centro y el maestro en la atención a las necesidades educativas especiales que se proponen

Adquirir recursos para promover la integración educativa de los estudiantes con dificultades.

Promover y colaborar en acciones dentro y fuera de la escuela, organizadas por familias, municipios y otras instituciones que tienen un impacto en la capacitación ciudadana

Identificar trastornos del sueño, nutrición, desarrollo psicomotor, atención y percepción auditiva y visual.

Saber de trabajar con otros profesionales de dentro y fuera de la atención del centro a cada estudiante, así como en la planificación de secuencias de aprendizaje y en la organización de las situaciones de trabajo en el aula y espacio de juego, identificando las peculiaridades del periodo 0-3 y del período 3-6

Asistir a las necesidades de los estudiantes y transmitir seguridad, tranquilidad y afecto.

Dominar las técnicas de observación y registro

Saber cómo analizar los datos obtenidos, comprender críticamente la realidad y elaborar un informe de conclusiones

Conocer los fundamentos de la expresión musical, plástica y corporal del currículum de esta etapa, así como las teorías sobre la adquisición y desarrollo del aprendizaje correspondiente

Saber cómo usar el juego como un recurso didáctico, así como también diseño de actividades de aprendizaje basadas en principios lúdicos

Conocer y aplicar los procesos de interacción y comunicación en el aula, así como dominar las habilidades y habilidades sociales necesarias para promover un clima que facilite el aprendizaje y la convivencia 
Relaciona la teoría y la práctica con el aula y el centro

Aprender a aprender

Resolver problemas y tomar decisiones de manera efectiva

Aplicar un pensamiento crítico, autocrítico, lógico y creativo

Trabajar en colaboración

Autonomía en el aprendizaje

Capacidad para análisis y síntesis

Capacidad para relacionarse positivamente con otras personas

Utilizar las herramientas básicas de las tecnologías de la información y comunicación (TIC) necesarias para el ejercicio de su profesión y para el aprendizaje a lo largo de lavida

Capacidad de organización y planificación

Capacidad de buscar y administrar información

Tabla 5. Competencias establecidas en la Guía Docente de la materia que fueron trabajadas durante la realización de esta actividad

Los contenidos abordados en las misiones también están vinculados totalmente con los contenidos programados en la Guía Docente de la materia, estos están recogidos en la Tabla 6.

\begin{tabular}{|c|}
\hline Contenidos abordados \\
\hline La psicomotricidad. Concepto y componentes. \\
\hline La educación a través de la acción motriz. \\
\hline Introducción a la organización anatómica y fisiológica en el niño. \\
\hline La motricidad fina y global, el esquema corporal, el equilibrio, el tono muscular y la coordinación. \\
\hline Las habilidades motrices básicas en la infancia \\
\hline
\end{tabular}

Tabla 6. Contenidos establecidos en la Guía Docente de la materia que fueron trabajados durante la realización de esta actividad

\section{RESULTADOS}

Para conocer los resultados obtenidos en esta experiencia didáctica se pasó un cuestionario anónimo para evaluar la satisfacción del alumnado sobre diferentes aspectos vinculados a la calidad docente. El cuestionario fue creado a través de google docs y enviado y distribuido a las cuentas oficiales de correo electrónico del alumnado por medio de la plataforma moodle. 
Los resultados se pasaron al programa estadístico SPSS para generar gráficos con las principales variables a estudio. En total, respondieron un total de 86 alumnos distribuidos de la siguiente forma (Tabla 7).

\begin{tabular}{|c|c|c|c|c|}
\hline$N=86$ & Media & Mediana & Mínimo & Máximo \\
\hline Edad & 22,34 & 21,50 & 19 & 34 \\
\hline Sexo & Mujer & Hombre & & \\
\hline$\%$ & 70,9 & 29,1 & & \\
\hline
\end{tabular}

En los siguientes gráficos se exponen los resultados relativos a las diferentes variables estudiadas.

\section{- Grado de acuerdo o desacuerdo con el ítem: Las clases eran aburridas}

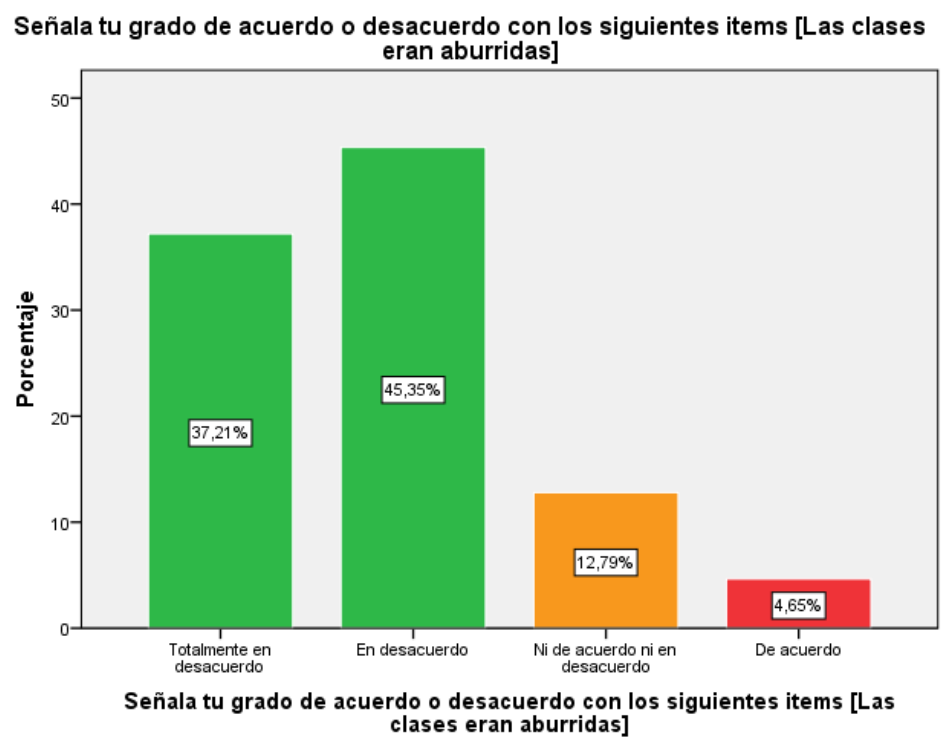

Gráfico 1. Grado de acuerdo o desacuerdo sobre el aburrimiento en las clases de las materias 
- Grado de acuerdo o desacuerdo con el ítem: Las clases eran motivantes

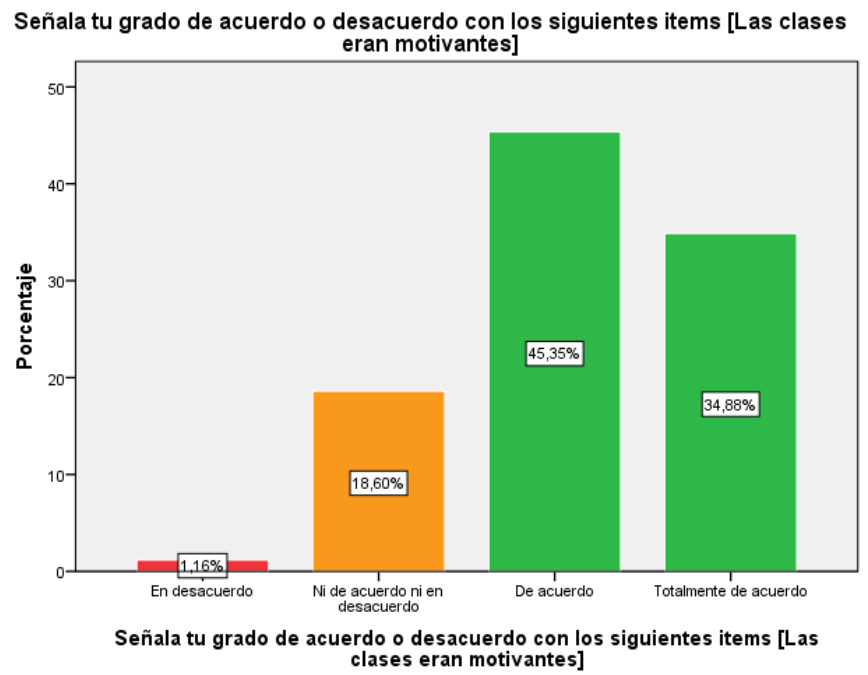

Gráfico 2. Grado de acuerdo o desacuerdo sobre la motivación en las clases de las materias

- Grado de acuerdo o desacuerdo con el ítem: Las actividades innovadoras mejoraron mi motivación hacia el estudio de los contenidos de la materia

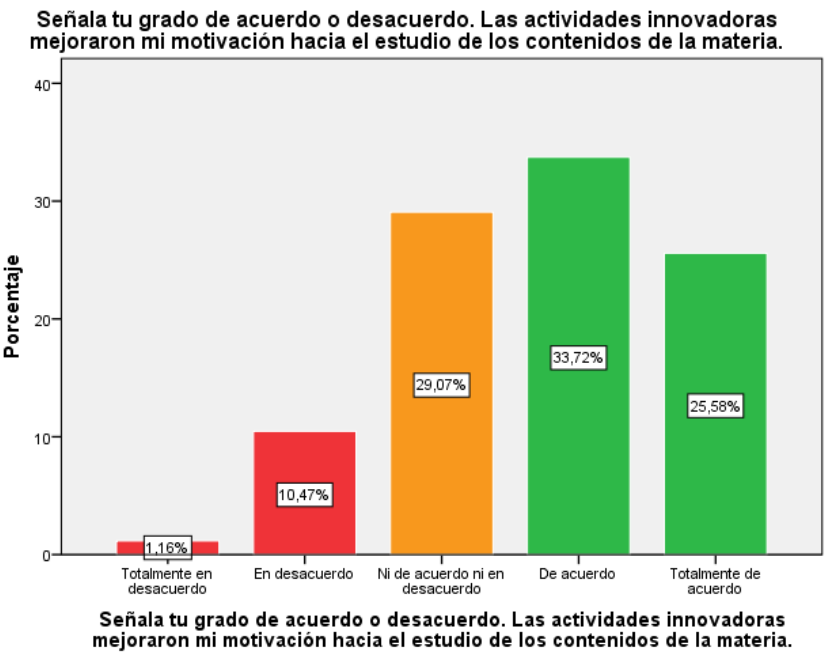

Gráfico 3. Grado de acuerdo o desacuerdo sobre las actividades innovadoras realizadas en el aula y la motivación hacia el estudio de la materia 
- Grado de acuerdo o desacuerdo con el ítem: Las actividades innovadoras mejoraron mi aprendizaje de los contenidos de la materia

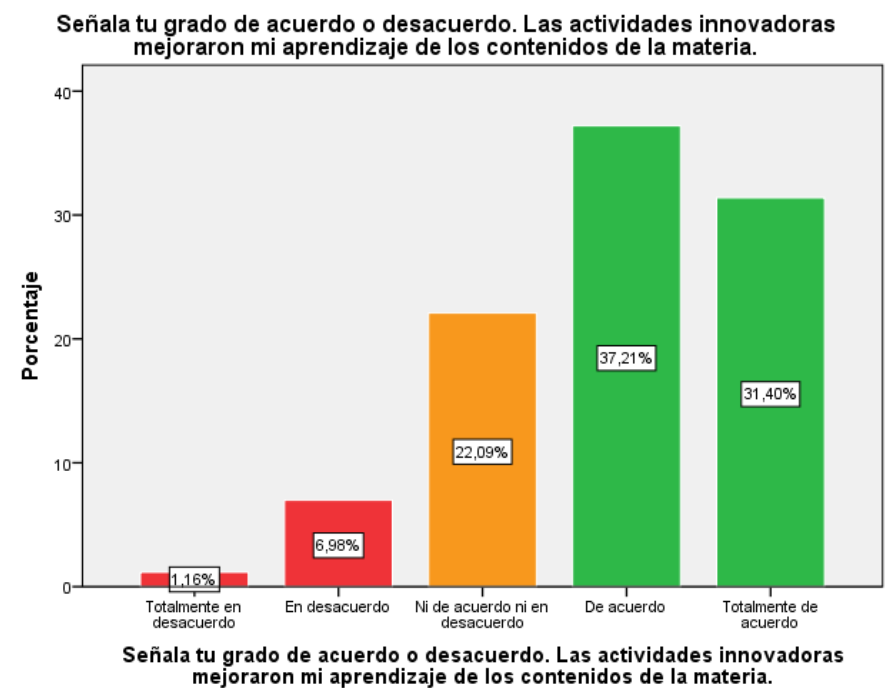

Gráfico 4. Grado de acuerdo o desacuerdo sobre las actividades innovadoras realizadas en el aula y la mejora del aprendizaje de la materia

\section{- Grado de satisfacción con el item: Herramientas innovadoras del docente}

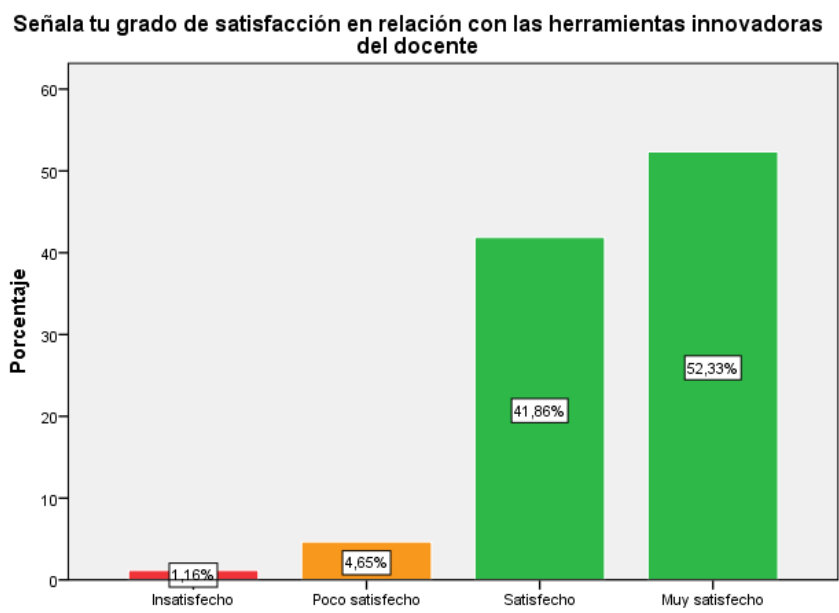

Señala tu grado de satisfacción en relación con las herramientas innovadoras del docente

Gráfico 5. Grado de satisfacción general sobre la innovación en el aula 


\section{CONCLUSIONES}

La aplicación en el aula universitaria de las tres experiencias didácticas basadas en la modificación de diferentes elementos de famosos programas de televisión produce una mayor satisfacción del alumnado en relación a su motivación y disfrute del estudio de los contenidos de la materia. El grado de satisfacción del alumnado es alto en las tres experiencias, por lo que podemos concluir que renovar el aula universitaria con nuevas metodologías y propuestas docentes puede enriquecer el disfrute del alumnado hacia los contenidos que se enseñan. Pese a no haber encontrado experiencias didácticas con estos programas de televisión, otros profesores que han gamificado sus aulas con otras propuestas y actividades confirman el éxito y disfrute para el alumnado de estas experiencias gamificadoras (Pisabarro Marrón \& Vivaracho Pascual, 2018). Incluso en algunos trabajos se confirma una mejora de rendimiento académico (Sanchez-Martin, Canada-Canada, \& Antonia Davila-Acedo, 2017).

\section{REFERENCIAS}

Arufe Giráldez, V. (2017a). FIRST DATES en el aula, una propuesta para el primer día de clase. Retrieved from http://www.victorarufe.com/firstdates/

Arufe Giráldez, V. (2017b). Máster chef para el aula. una actividad con la que disfrutará y $\begin{array}{llll}\text { aprenderá } & \text { el } & \text { alumnado. } & \text { Retrieved }\end{array}$ http://www.victorarufe.com/2017/12/11/m\%C3\%A1ster-chef-para-el-aula-una-actividad-conla-que-disfrutar\%C3\%A1-y-aprender\%C3\%A1-el-alumnado/ Arufe Giráldez, V. (2018, -04-14T05:00:00CEST). Ellos son los mejores. La Voz De Galicia Retrieved from https://www.lavozdegalicia.es/noticia/yes/2018/04/14/mejores/0003_201804SY14P2991.htm Contreras Espinosa, R. S., \& Eguia, J. L. (2016). Gamificación en aulas universitarias\&nbsp;. Barcelona: Universidad Autónoma de Barcelona. 
Deterding, S., Khaled, R., Nacke, L. E., \& Dixon, D. (2011). Gamification : Toward a definition. Chi, Retrieved from /paper/Gamification-\%3A-Toward-a-Definition-DeterdingKhaled/84fe3e41153d73dd0675ae14501a497c22b2a63f

Mora Teruel, F. (2014). Neuroeducación. Madrid: Alianza.

Pisabarro Marrón, A. M., \& Vivaracho Pascual, C. E. (2018). Gamificación en el aula: Gincana de programación\&nbsp; ReVisión, 11(1), 85-93.

Sanchez-Martin, J., Canada-Canada, F., \& Antonia Davila-Acedo, M. (2017). Just a game? gamifying a general science class at university collaborative and competitive work implications. Thinking Skills and Creativity, 26, 51-59. 10.1016/j.tsc.2017.05.003 